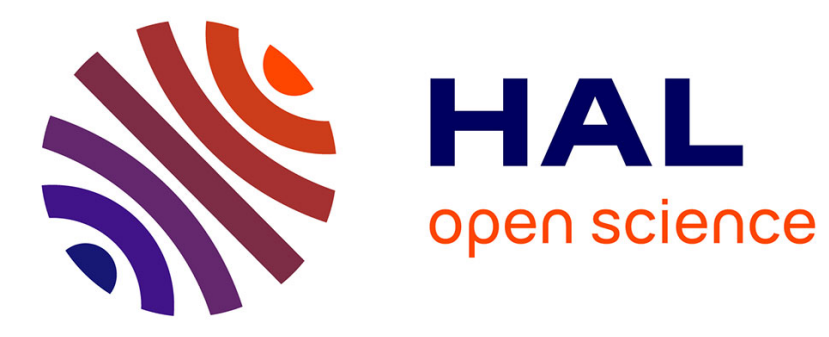

\title{
Does Preferred Seat Pan Inclination Minimize Shear Force?
}

Xuguang Wang, Michelle Cardosso, Ilias Theodorakos, Georges Beurier

\section{To cite this version:}

Xuguang Wang, Michelle Cardosso, Ilias Theodorakos, Georges Beurier. Does Preferred Seat Pan Inclination Minimize Shear Force?. IEA\&apos;18, 20th Congress of the International Ergonomics Association, Aug 2018, Florence, Italy. 7p, 10.1007/978-3-319-96077-7_30 . hal-03236053

\section{HAL Id: hal-03236053 https://hal.science/hal-03236053}

Submitted on 26 May 2021

HAL is a multi-disciplinary open access archive for the deposit and dissemination of scientific research documents, whether they are published or not. The documents may come from teaching and research institutions in France or abroad, or from public or private research centers.
L'archive ouverte pluridisciplinaire HAL, est destinée au dépôt et à la diffusion de documents scientifiques de niveau recherche, publiés ou non, émanant des établissements d'enseignement et de recherche français ou étrangers, des laboratoires publics ou privés. 
See discussions, stats, and author profiles for this publication at: https://www.researchgate.net/publication/326879860

Does Preferred Seat Pan Inclination Minimize Shear Force?: Volume V: Human Simulation and Virtual Environments, Work With Computing Systems (WWCS), Process Control

Chapter · January 2019

DOI: 10.1007/978-3-319-96077-7_30

4 authors, including: 


\title{
Does preferred seat pan inclination minimize shear force?
}

\author{
Xuguang Wang
kos $^{[0000-0001-9181-6045]}$, Michelle Cardosso-0002-7558-7286]
, Georges Beurier
$[0000-00003-2422-6365]$ \\ Univ Lyon, Université Claude Bernard Lyon 1, IFSTTAR, LBMC UMR_T9406, F69675, \\ Lyon, France \\ xuguang.wangdifsttar.fr
}

\begin{abstract}
Past biomechanical studies on seated postures showed that effects of seat parameters, such as seat pan angle, back angle and friction coefficient, on muscle activities, shear force between buttocks and seat and spinal loads are complex. Reducing all these biomechanical loads at the same time may not be possible. Lowered muscle activation may require higher frictional shear force. It is interesting to investigate how people behave compared to biomechanical simulations. In this paper, the question whether sitters prefer a seat pan angle for reducing shear force was investigated using the data collected from a multiadjustable experimental seat. Two imposed seat pan angles $\left(\right.$ A_SP $\left.=0^{\circ}, 5^{\circ}\right)$ and one self-selected were tested for two backrest angles (A_SB $=10^{\circ}, 20^{\circ}$, from the vertical). A flat seat pan surface was used. Other seat parameters such as seat height, length and position of three back supports were defined with respect to each participant's anthropometry. As expected, results showed that shear force increased with backrest recline and decreased with seat pan recline. No significant difference in self-selected seat pan angle was found between two backrest angles. An average of $6.2^{\circ}\left( \pm 3^{\circ}\right)$ was observed. The lowest shear was observed for the condition of self-selected seat pan angle, supporting the idea that seat pan should be oriented to minimize shear force. However, self-selected angle did not completely remove the shear. A zero shear would require a more reduced trunk-thigh angle, suggesting a minimum trunk-thigh angle should also be maintained.
\end{abstract}

Keywords: Seating, Biomechanics, Shear force, Discomfort, Airplane passenger.

\section{Introduction}

Reed et al. (2000) suggested both pressure and shear force on the seat contact surface affect sitting discomfort [4]. It is generally recommended that peak pressure should be reduced and located at the area of the ischial tuberosities. Though large differences in pressure distribution and sensitivity among individuals make specifying a quantitative "optimal" pressure distribution difficult, Mergl et al (2005) tended to determine ideal distribution [3]. It is also recommended that surface shear on the seat cushion should be minimized by changing the cushion angle and/or contouring the cushion. Goossens and Snijders (1995) theoretically and experimentally investigated the relationship 
between seat and backrest inclinations for removing shear force [2]. They found that a fixed inclination between seat and backrest should be chosen between $90^{\circ}$ and $95^{\circ}$. Rasmussen and his colleagues developed a computational musculoskeletal seated human model and used it for investigating the effects of backrest inclination, seat pan inclination and seat surface friction coefficient on muscle activity, shear force in the buttocks and spinal loads by simulation $[5,6,7]$. It was shown that forward seat-pan inclination (up to about $15^{\circ}$ ) reduces the spinal-joint L4-L5 compression force for a fixed backrest inclination of $10^{\circ}$ backward [7]. Muscle relaxation and shear force reduction may be conflicting [5]. A linear relationship between seat pan and backrest angles was found when both shear force and muscle activity were minimized [6]. An angle of less than $90^{\circ}$ between backrest and seat pan was found without considering spinal load reduction. This seems quite unrealistic when comparing with existing aircraft or office seats which have a more opened angle between backrest and seat pan. By simulation, Rasmussen and his colleagues showed that the effects of seat parameters (seat pan and backrest angles, coefficient of friction) on muscle activities, shear force and spinal loads are complex. To date, few researchers have performed a parametric study of seat parameters on sitting discomfort. More specifically, few have verified experimentally whether people prefer a seat pan angle minimizing the shear force as it may be conflicting with other criterions such as reducing muscle activities and spinal joint load. This was one of the research questions we investigated experimentally using a newly built multi-adjustable experimental seat [1] in a research program for improving the comfort of economy class aircraft seats.

\section{Materials and methods}

\subsection{Participants}

Thirty-six participants (18 males, 18 females), aged from to 19 to 56 years old, were recruited based on their body mass index (BMI) (healthy 18.5-25, obese >30) and stature (small, medium and tall). Three stature groups were formed: $154-157 \mathrm{~cm}, 162-$ $166 \mathrm{~cm}$ and $170-175 \mathrm{~cm}$ for females; $168-171 \mathrm{~cm}, 176-180 \mathrm{~cm}$ and $185-190 \mathrm{~cm}$ for males. A total of 12 groups were formed after considering sex, stature and BMI (3 individuals per group). Prior to the experiment, participants were screened using a health questionnaire. Participants who experienced any back injury or pain in the previous 3-months were excluded. The experimental protocol was approved by IFSTTAR (French Institute of Science and Technology for Transport, Development and Networks) ethics committee and informed consent was given prior to experiment.

\subsection{Experimental seat}

The data was collected through use of the multi-adjustable experimental seat recently developed at IFSTTAR. The experimental seat had thirteen adjustable parameters directly controlled by a computer. Adjustable features included: fore-aft (x) and vertical position $(\mathrm{z})$ of the foot support, seat pan and three back supports; rotational angle of the seat pan, backrest and global inclination of the whole experimental seat. Two 
armrests were also available and adjusted manually. Force sensors were mounted to measure contact forces in $\mathrm{xz}$ plane on the foot support, seat pan, three back supports and two armrests. The seat pan surface was composed of a matrix of 52 cylinders, each with a freely rotatable circular flat head of $60 \mathrm{~mm}$ in diameter. Each cylinder was equipped with a tri-axial force sensor, enabling the measurement of both normal and tangential forces. The height of each cylinder was adjustable with a maximum stroke length of $40 \mathrm{~mm}$ and pressure distribution could be controlled by changing seat surface. Pressure distribution on the seat pan surface was controlled using a uniform coupling law relating normal force and position for each cylinder. The coupling law enabled us to distribute normal contact force as uniformly as possible among the 52 cylinders (given the maximum displacement of the cylinders). A more detailed description of the experimental seat can be found in [1].

\subsection{Experimental conditions and procedure}

Participants were instructed to test a total of 40 seat configurations that simulated an economy class airplane seat. The H-point location of an existing airplane seat was used to define the $\mathrm{x}$ position of the middle back support. Two backrest angles (A_SB) from the vertical $\left(10^{\circ}, 20^{\circ}\right)$ and three seat pan angles (A_SP $=0^{\circ}, 5^{\circ}$, preferred) were used to define 6 different A_SP/A_SB combinations covering the range of variation of airplane eco-class passenger seats. For each combination, five conditions were tested successively. The first one, called 'reference position' with a flat seat pan surface, was used to determine seat pan length, foot support height and armrests position for each participant. The three backrest panels were positioned at specific anatomical points (occipital bone, T9 and L3). Their position in $\mathrm{x}$ was fixed at $135 \mathrm{~mm}$ in the seat back LCS. The seat pan length (X_SP_L, Fig. 1a) was fixed until there was approximately $70 \mathrm{~mm}$ (hand width) between the popliteal (behind the knee) and the front of the seat pan. Participants were asked to keep their back in contact with the lower and middle supports. The foot support was adjusted (Z_FS, Fig. 1a) until the knees were set at approximately 90 degrees. Participants were also asked to place rectangular foam of $100 \mathrm{~mm}$ (in thickness) between the knees to reduce postural variation (Fig. 1b). The armrests were self-positioned by subjects. Once participants were fitted to the seat, they were instructed to step off the experimental seat to zero all the force sensors. Then, they were asked to reposition themselves back on the experimental seat and look forward without use of the upper support. Measurements were recorded at a rate of $20 \mathrm{~Hz}$ for 1.25 seconds. The preferred seat pan angle was self-selected by participants for the reference position and kept unchanged for the four other test conditions with a fixed backrest angle. Four others conditions were aimed to study the effects of posture (relaxing with use of head support, looking forward without using head support) and pressure distribution on seat. In this study, only data from the reference position were used. 


\subsection{Data processing and analysis}

The medians of the measurements of each trial were calculated at first. Inconsistent trials due to either measurement or manipulation errors were eliminated at first. Then, multifactor ANOVAs were performed using STATGRAPHICS Centurion XVII. Effects of independent variables were considered 'significant' when $\mathrm{p}<0.05$.
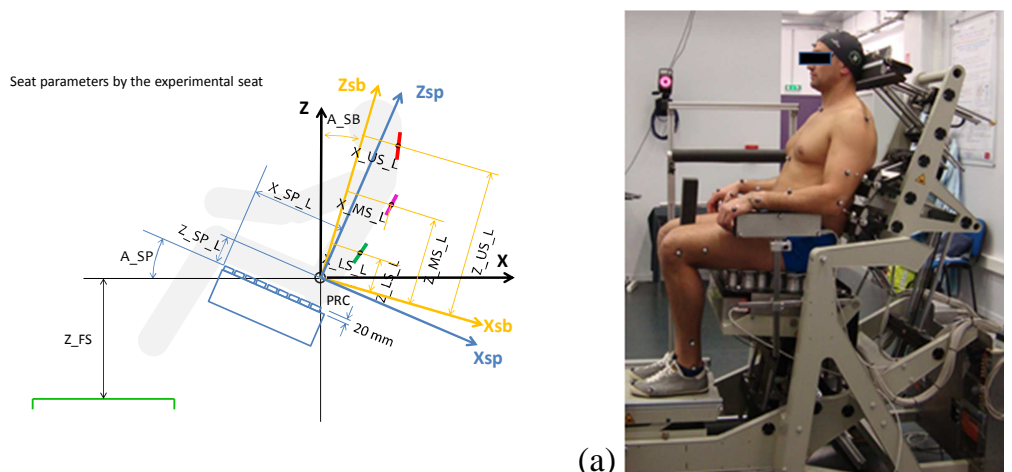

(b)

Fig. 1. Multi-adjustable seat. Definition of main adjustable seat parameters (a) and illustration of a participant sitting on the experimental seat (b)

\section{$3 \quad$ Results}

To answer the research question whether participants preferred a seat pan angle which reduces shear force, the self-selected seat pan angle and global shear force applied on the seat pan surface were analyzed and results are shown in Table 1 and Table 2. Preferred seat pan angles (A_SP) were $5.74^{\circ}$ and $6.67^{\circ}$ respectively for a seat back angle (A_SB) of $10^{\circ}$ and $20^{\circ}$, showing that a slightly more reclined seat pan was selected for a more reclined backrest though no significant difference was observed between two backrest angles. The shear forces corresponding to the preferred seat pan angle are compared with two other imposed seat pan angles in Table $\mathbf{2}$ for two back angles. Both A_SP and A_SB significantly affected the shear force, which increased when increasing A_SB and lowering A_SP. As expected, the lowest shear force was observed for self-selected seat pan angle. The shear forces corresponding to preferred seat pan angle were respectively $4.61 \%$ and $6.19 \%$ of the body weight.

Table 1. Self-selected seat pan angle in degrees for seat back angle (A_SB) being fixed at $10^{\circ}$ and $20^{\circ}$ from the vertical backward

\begin{tabular}{lllllll}
\hline A_SB & Count & Average & SD & Minimum & Maximum & Range \\
\hline $10^{\circ}$ & 34 & 5.74 & 3.05 & 0.26 & 14.02 & 13.76 \\
$20^{\circ}$ & 36 & 6.67 & 3.01 & 0.45 & 13.84 & 13.39 \\
Total & 70 & 6.22 & 3.05 & 0.26 & 14.02 & 13.76 \\
\hline
\end{tabular}


Table 2. Means and standard deviations of the normalized shear force applied at the seat pan surface when varying both seat pan angle (A_SP) and seat back angle (A_SB). PR is self-selected. Forces are in percentage of body weight. A negative force means the shear force applied on the seat is directed forward.

\begin{tabular}{lllllll}
\hline & A_SB=10 & \multicolumn{3}{c}{ A_SB=20 } & \multicolumn{3}{c}{ Total } \\
A_SP & Mean & SD & Mean & SD & Mean & SD \\
\hline $0^{\circ}$ & -8.56 & 1.78 & -10.57 & 1.79 & -9.58 & 2.05 \\
$5^{\circ}$ & -4.73 & 2.17 & -6.92 & 1.93 & -6.38 & 2.20 \\
PR & -4.61 & 2.97 & -6.19 & 3.01 & -5.42 & 3.07 \\
Total & -5.97 & 2.98 & -7.51 & 2.67 & -6.94 & $2.88^{\text {A_SB***,A_SP*** }}$ \\
\hline
\end{tabular}

$* \mathrm{P}<0.05, * * \mathrm{P}<0.01, * * * \mathrm{P}<0.001$

\section{Discussion and conclusion}

In this paper, the question whether sitters prefer a seat pan angle for reducing shear force was investigated using the data collected from a multi-adjustable experimental seat. As expected, lower shear force at the buttock seat contact surface was observed when participants were allowed to self-select their preferred seat pan angle. Compared to a horizontal seat pan, shear force was reduced from $8.56 \%$ to $4.61 \%$ and from $10.57 \%$ to $6.19 \%$ of body weight on average respectively for a seat back angle of $10^{\circ}$ and $20^{\circ}$. Our observations support the general idea that a reduced shear force is preferred.

Preferred seat pan angles were about $6^{\circ}$ and $7^{\circ}$ for a back angle of $10^{\circ}$ and $20^{\circ}$. Compared to the recommendations by Rasmussen et al [6] from simulations for minimizing both muscle activities and shear force, self-selected seat pan angles observed in the present study were much less reclined (backward), rather in agreement with the requirement of a minimum angle of $105^{\circ}$ between seat pan and back (trunk-thigh angle) suggested by Keegan in 1953 [8]. Keegan considered that a good lumbar curvature could not be preserved with a trunk-thigh angle smaller than $105^{\circ}$. To fully remove shear force, a more reclined seat pan would be required with a more reduced trunk-thigh angle, as already predicted and observed by Goossens and Snijders [2]. This was not preferred by participants.

In the present study, a multi-adjustable experimental seat was used. Except for seat pan angle, other seat parameters such as seat height, seat pan length, position of the lumbar and thoracic supports were defined with respect to each participant's anthropometry. Thus their possible interactions with seat pan inclination were eliminated. For example, when changing seat pan angle, seat height was also adapted to avoid high compression of the posterior thigh. Results obtained in the present study can hardly be obtained without using a multi-adjustable experimental seat. The main limitation is also related to the use of an experimental seat. The preferred seat pan angle was obtained with a flat seat surface without any soft cushion. The backrest was sim- 
plified by three supports. A same initial seat pan angle was not imposed before asking participants to select their preferred seat pan angle. This may explain, at least partly, the large variation of self-selected angles. In a follow-up study [9], it was observed that preferred seat pan angle depended on initial seat pan inclination. An initially small seat pan angle would lead to a less reclined self-selected seat angle, suggesting that a range of seat pan orientation would be preferred.

In conclusion, sitters preferred a seat pan inclination with a reduced shear force, while a minimum angle of trunk thigh angle should be kept as suggested by Keagan [8].

\section{Acknowledgement}

The work is partly supported by Direction Générale de l'Aviation Civile (project $\left.n^{\circ} 2014930818\right)$.

\section{References}

1. Beurier, G., Cardoso, M., and Wang, X.: A New Multi-Adjustable Experimental Seat for Investigating Biomechanical Factors of Sitting Discomfort. SAE Technical Paper. 201701- 1393, doi:10.4271/2017-01- 1393 (2017).

2. Goossens, R.H.M., Snijders, C.J.: Design criteria for the reduction of shear forces in beds and seats. Journal of Biomechanics 28(2), pp.225-230 (1995).

3. Mergl, C., Klendauer, M., Mangen, C., Bubb, H.: Predicting long term riding comfort in cars by contact forces between human and seat. SAE Technical Paper $\mathrm{N}^{\circ}$ 2005-01-2690 (2005).

4. Reed, M. P.: Survey of auto seat design recommendations for improved comfort. Michigan Transportation Research Institute (UMTRI) (2000).

5. Rasmussen, J., de Zee, M., Tørholm, S.: Muscle relaxation and shear force reduction may be conflicting: a computational model of seating. SAE Technical Paper 2007-01-2456 (2007). DOI: https://doi.org/10.4271/2007-01-2456.

6. Rasmussen, J., de Zee, M.: Design optimization of airline seats. SAE Technical Paper 2008-01-1863 (2008).

7. Rasmussen, J., Tørholm, S., de Zee, M.: Computational analysis of the influence of seat pan inclination and friction on muscle activity and spinal joint forces. International Journal of Industrial Ergonomics 39, 52-57 (2009).

8. Keegan, J.J.: Alterations of the lumbar curve related to posture and seating. The Journal of Bone and Joint Surgery 35A, 589-603 (1953).

9. Theodorakos, I., Savonnet, L., Beurier, G., Wang, X.: Can computationally predicted internal loads be used to assess sitting discomfort? Preliminary results. IEA 2018, Florence, Italy (2018). 